\title{
Flexible academic support to enhance student retention and success
}

Hendrik van der Sluis

Steve May

Academic Development Centre

Lorraine Allibone

LSHTM

What can higher education contribute to improving social mobility in the UK? HEA conference, 26 and 27 March 2013

Available at: goo.gl/MkfRI 


\section{Introduction and context}

\section{Changing HE Landscape}

Widening participation in particular means that students have more diverse expectations, learning styles and needs (e.g. BIS, 2010; Milburn, 2009

Increased focussed on employability and the development of transferable skills(e.g. Browne, 2010; Leitch, 2006; Pegg et al, 2002)

This is being addressed at one institution through dialogue with students and their inclusion as practitioners in the provision of skills development (Myers \& Gibsen, 2010)

Increased fees for students at English universities, attainment and employability have become key measure for students and university reputation and status 


\section{Introduction and context}

\section{Kingston University}

The university in South West London has 5 faculties across 4 campuses. It has a high proportion of students from non traditional backgrounds.

24500 students of whom 20000 are undergraduates.

$50 \%$ of students from ethnic minorities

$39 \%$ of young first UG entrants with parents from lower socioeconomic classes (WP) 


\section{Development of ASC}

\section{Academic Skills Centres}

Developed as part of the Kingston University Teaching and Learning strategy (2009)

Diversity of student population -> diverse needs -> diversified approach

Campus based centres - different identities, faculty based

Provision through consultation (academic advisor) and online materials

Delivery and tools informed by dialogue with students, partly designed by students

Academic mentors mixture of faculty staff and $>2$ nd students

Support offered include: essay and report writing, referencing, mathematics and programming 


\section{Evaluation of the ASC}

\section{Attendance data and SITS}

Attendance data (2011-12)

Supplemented with student information records (SITS)

\section{Main research questions:}

1. To what extent do the ASCs reach out to students from non traditional backgrounds?

2. Which skills are sought by students from traditional and non-traditional backgrounds?

3. Do the ASCs enhance progression and attainment? 


\section{Demographics of ASC (non)attendees}

\begin{tabular}{llrrrr} 
& & \multicolumn{2}{c}{ ASC attendees } & \multicolumn{2}{c}{ Non ASC attendees } \\
\hline \multirow{2}{*}{ Mode } & Full time & 1552 & $95 \%$ & 20038 & $83 \%$ \\
\cline { 2 - 6 } & Part time & 77 & $5 \%$ & 4125 & $17 \%$ \\
\hline \multirow{2}{*}{ Level } & Sub degree & 108 & $7 \%$ & 2912 & $12 \%$ \\
\cline { 2 - 6 } & Hons degree & 1238 & $76 \%$ & 15398 & $65 \%$ \\
\cline { 2 - 6 } & Masters degree & 280 & $17 \%$ & 5445 & $23 \%$ \\
\hline \multirow{2}{*}{ Gen on entry } & Under 21 & 881 & $54 \%$ & 12995 & $54 \%$ \\
\cline { 2 - 6 } & Over 21 & 748 & $46 \%$ & 11168 & $46 \%$ \\
\cline { 2 - 6 } & Female & 1095 & $67 \%$ & 11682 & $48 \%$ \\
\cline { 2 - 6 } & Male & 534 & $33 \%$ & $52 \%$ \\
\hline
\end{tabular}




\section{Demographics of ASC (non)attendees}

ASC attendees Non ASC attendees

\begin{tabular}{|c|c|c|c|c|c|}
\hline \multirow{2}{*}{$\begin{array}{l}\text { Ethnic background } \\
\text { where known }\end{array}$} & BME & 977 & $61 \%$ & 11749 & $49 \%$ \\
\hline & White & 615 & $39 \%$ & 11989 & $51 \%$ \\
\hline \multirow{2}{*}{$\begin{array}{l}\text { Socio economic class } \\
\text { of young students (NS- } \\
\text { SEC) }\end{array}$} & $1-3$ & 285 & $59 \%$ & 4950 & $59 \%$ \\
\hline & 4-7 (WP) & 200 & $41 \%$ & 3394 & $41 \%$ \\
\hline \multirow{5}{*}{$\begin{array}{l}\text { Neighbourhood quintile } \\
\text { of young students } \\
\text { (Polar } 3 \text { ) }\end{array}$} & Q1 (WP) & 39 & $5 \%$ & 633 & $5 \%$ \\
\hline & Q2 (WP) & 77 & $10 \%$ & 1166 & $10 \%$ \\
\hline & Q3 & 185 & $25 \%$ & 2758 & $24 \%$ \\
\hline & Q4 & 184 & $25 \%$ & 2918 & $25 \%$ \\
\hline & Q5 & 254 & $34 \%$ & 4221 & $36 \%$ \\
\hline
\end{tabular}




\section{Skills sought}

\begin{tabular}{|c|c|c|c|c|c|c|c|c|c|}
\hline \multirow[b]{2}{*}{ Skills assessed } & \multirow[b]{2}{*}{$\begin{array}{l}\text { visit } \\
\text { s }\end{array}$} & \multicolumn{2}{|c|}{ Eth group } & \multicolumn{2}{|c|}{ Age group } & \multicolumn{2}{|c|}{ NS-SEC (young) } & \multicolumn{2}{|c|}{ Quin group (young) } \\
\hline & & BME & White & $\mathbf{m}$ & $\mathbf{y}$ & $1-3$ & 4-7 (WP) & $1-2$ (WP) & $3-5$ \\
\hline Referencing & 2418 & $59 \%$ & $41 \%$ & $47 \%$ & $53 \%$ & $58 \%$ & $42 \%$ & $13 \%$ & $87 \%$ \\
\hline Grammar and punctuation & 1199 & $57 \%$ & $43 \%$ & $51 \%$ & $49 \%$ & $59 \%$ & $41 \%$ & $13 \%$ & $87 \%$ \\
\hline Other English & 2078 & $60 \%$ & $40 \%$ & $53 \%$ & $47 \%$ & $56 \%$ & $44 \%$ & $15 \%$ & $85 \%$ \\
\hline Mathematics & 173 & $68 \%$ & $32 \%$ & $46 \%$ & $54 \%$ & $45 \%$ & $55 \%$ & $35 \%$ & $65 \%$ \\
\hline Computing & 313 & $75 \%$ & $25 \%$ & $37 \%$ & $63 \%$ & $47 \%$ & $53 \%$ & $48 \%$ & $52 \%$ \\
\hline General discussion & 475 & $68 \%$ & $32 \%$ & $55 \%$ & $45 \%$ & $55 \%$ & $45 \%$ & $16 \%$ & $84 \%$ \\
\hline Employability & 71 & $67 \%$ & $33 \%$ & $40 \%$ & $60 \%$ & $45 \%$ & $55 \%$ & $14 \%$ & $86 \%$ \\
\hline Dyslexia and disability & 63 & $48 \%$ & $52 \%$ & $54 \%$ & $46 \%$ & $50 \%$ & $50 \%$ & $30 \%$ & $70 \%$ \\
\hline Other skills & 192 & $70 \%$ & $30 \%$ & $38 \%$ & $62 \%$ & $61 \%$ & $39 \%$ & $13 \%$ & $87 \%$ \\
\hline Sum of Skills All visits & 3547 & $59 \%$ & $41 \%$ & $51 \%$ & $49 \%$ & $56 \%$ & $44 \%$ & $15 \%$ & $85 \%$ \\
\hline
\end{tabular}


Impact on progression First year progression rates of ASC attendees and non attendees (Full time, Honours degree only)

ASC attendees

Non ASC attendees

\begin{tabular}{llllll}
\hline & & $\mathrm{n}$ & progressed & $\mathrm{n}$ & progressed \\
\hline \multirow{2}{*}{ All } & & 540 & $91 \%$ & 5905 & $78 \%$ \\
& Female & 351 & $92 \%$ & 3033 & $84 \%$ \\
\cline { 2 - 6 } & Male & 189 & $89 \%$ & 2872 & $71 \%$ \\
\hline Age on entry & Under 21 & 341 & $91 \%$ & 4235 & $77 \%$ \\
\cline { 2 - 6 } & Over 21 & 199 & $90 \%$ & 1670 & $81 \%$ \\
\hline $\begin{array}{l}\text { Ethnic background where } \\
\text { known }\end{array}$ & BME & 288 & $88 \%$ & 2821 & $72 \%$ \\
\cline { 2 - 6 } & White & 233 & $94 \%$ & 2956 & $84 \%$ \\
\hline $\begin{array}{l}\text { SEC (NS-SEC) of young } \\
\text { students where known }\end{array}$ & $1-3$ & 105 & $90 \%$ & 1658 & $79 \%$ \\
\cline { 2 - 6 } & $4-7$ (WP) & 71 & $93 \%$ & $73 \%$ \\
\end{tabular}




\section{Impact on Attainment, faculty of Business and Law, 'Business Environment'}

\section{"Business Environment" module attainment}

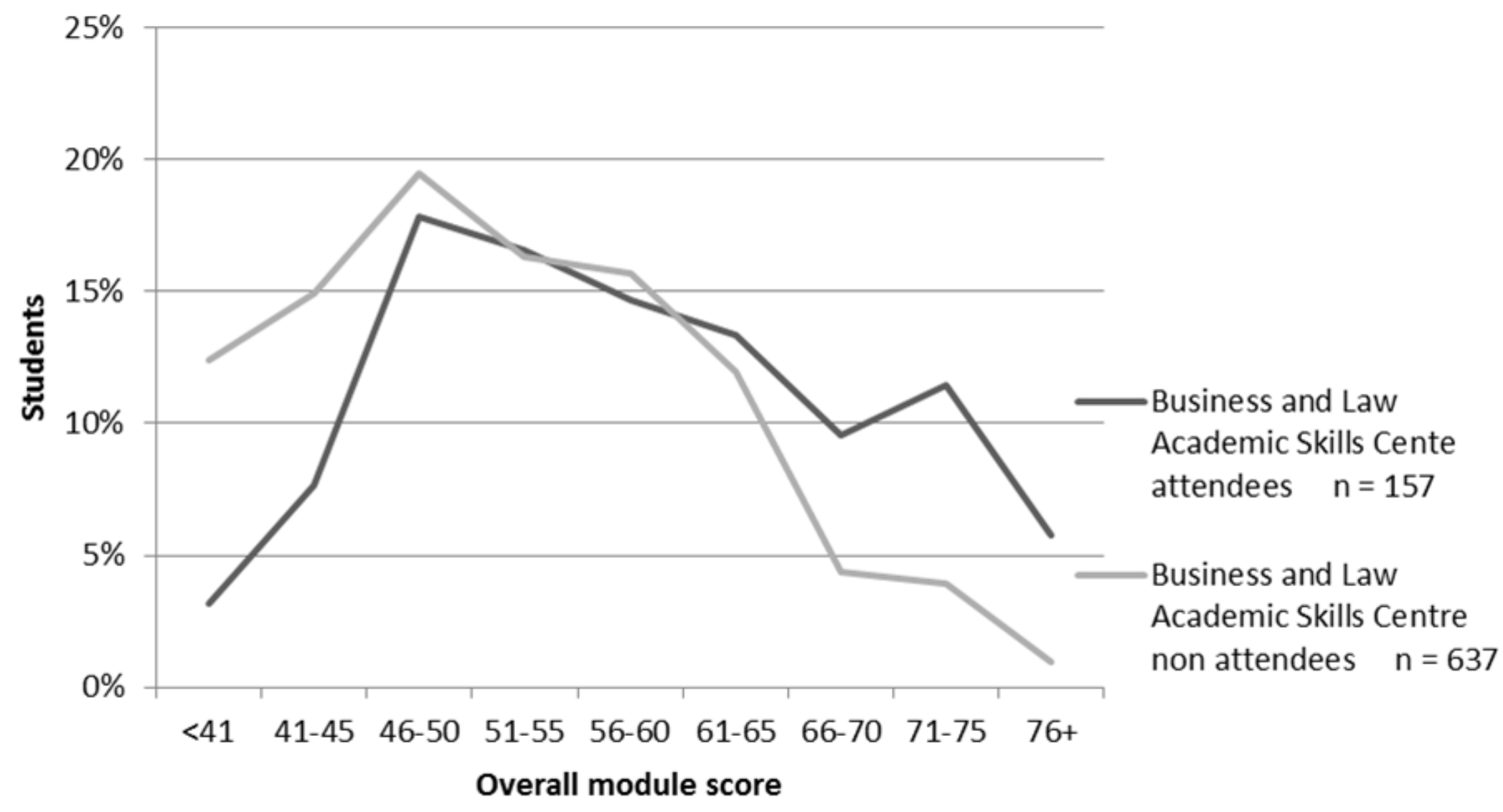




\section{Summary \& Discussion}

\section{Attendance}

Visited by full time, undergraduate, with higher representation of female and BME students

Skills most sought are around English and academic writing

Significant relationship between visiting and likelihood of progression

Positive effect on attainment

Discussion and future direction

Voluntary attendance more self aware and motivated?

Changing landscape, increased competition, raising entry criteria 